\title{
Synthesising Research Results
}

\author{
Barbara Kitchenham
}

I am currently working on the Evidence-Based Software Engineering (EBSE) project (EP/C51839X/1) awarded by the UK Engineering and Physical Sciences Research Council. We are investigating how readily the concept of evidence-based practice can be adapted to Software Engineering. Evidence-based practice relies on research synthesis, aggregating and analysing relevant 'primary studies' by using the methodology of systematic literature reviews. Much of the original impetus came from medical research and emphasised synthesis of high-quality quantitative experiments (i.e. double-blind randomised controlled trials) using statistical metaanalysis.

As part of our research we are reviewing the use of evidence-based practice in social science related domains which have empirical practices that are more similar to Software Engineering than those of medicine [1] Research originating in these disciplines has identified sound methods for synthesising evidence from different types of studies, including qualitative studies [2].

In particular, research synthesis of mixed study types needs to:

- Assess the quality of each primary study using criteria appropriate to the study type.

- Use study quality and study type as "moderator variables" to assist interpreting data.

- Synthesise studies based on different methods separately.

This approach can be used for research synthesis of systematic reviews including both qualitative and quantitative primary studies. For example, a cross-study synthesis of .a quantitative systematic review and a qualitative systematic review should assess the extent of agreement between the reviews. Interventions considered important in both types of survey are likely to be the most beneficial.

\section{References}

1. David Budgen, Stuart Charters, Mark Turner, Pearl Brereton, Barbara Kitchenham \& Steve Linkman. (2006) Transferring the Evidence-based paradigm to Software Engineering. Proceedings of the WISER workshop, ICSE 2006, ACM Press, pp 7-13.

2. Mark Petticrew and Helen Roberts. Systematic Reviews in the Social Sciences. A Practical Guide. Blackwell Publishing Ltd. 2006. 